\title{
Long non-coding RNA MEG3 contributes to cisplatin-induced apoptosis via inhibition of autophagy in human glioma cells
}

\author{
BINBIN MA ${ }^{1 *}$, ZEBIN GAO $^{2 *}$, JIACHENG LOU $^{1}$, HONGQIANG ZHANG $^{1}$, \\ ZHONGBO YUAN ${ }^{1}$, QIONG WU ${ }^{3}$, XINYU $\mathrm{LI}^{4}$ and BO ZHANG ${ }^{1}$

\begin{abstract}
Departments of ${ }^{1}$ Neurosurgery and ${ }^{2}$ Clinical Laboratory, The Second Affiliated Hospital of Dalian Medical University, Dalian, Liaoning 116023; Departments of ${ }^{3}$ Neurology and ${ }^{4}$ Endocrinology,

Dalian Municipal Central Hospital Affiliated to Dalian Medical University, Dalian, Liaoning 116033, P.R. China
\end{abstract}

Received May 31, 2016; Accepted April 6, 2017

DOI: $10.3892 / \mathrm{mmr} .2017 .6897$

\begin{abstract}
Long non-coding RNAs (lncRNAs) function as oncogenes or tumor suppressors, and are involved in mediating tumorigenesis and resistance to chemotherapy by altering the expression of genes at various levels. Accumulating evidence suggests that the maternally expressed gene 3 (MEG3) lncRNA serves an important role in a number of cancers. However, its functional role in mediating cisplatin-induced apoptosis of glioma cells is unknown. To investigate the role of MEG3, the mRNA levels of MEG3 under cisplatin treatment were investigated by reverse transcription-quantitative polymerase chain reaction, and the cell viability and apoptosis were examined by MTT assay, and flow cytometry analysis and western blotting, respectively. The results demonstrated that MEG3 expression levels were increased in U87 cells following cisplatin treatment. Elevated MEG3 by lentiviral transfection enhanced the chemosensitivity of U87 cells to cisplatin, whereas knockdown of MEG3 expression by small interfering RNA transfection increased the resistance of U87 cells to cisplatin. Subsequent mechanistic studies revealed that MEG3 eliminated autophagy induced by cisplatin. Decreased MEG3-induced autophagy improved the chemosensitivity of U87 cells to cisplatin. The results present a novel therapeutic strategy for the treatment of patients with glioblastoma multiforme.
\end{abstract}

Correspondence to: Professor Bo Zhang, Department of Neurosurgery, The Second Affiliated Hospital of Dalian Medical University, 467 Zhongshan Road, Dalian, Liaoning 116023, P.R. China

E-mail: zhangbodl@126.com

Professor Xinyu Li, Department of Endocrinology, Dalian Municipal Central Hospital Affiliated to Dalian Medical University, 42 Xuegong Street, Dalian, Liaoning 116033, P.R. China

E-mail: dlxinyuli1969@163.com

*Contributed equally

Key words: maternally expressed gene 3, apoptosis, autophagy, cisplatin, glioma cell

\section{Introduction}

Glioblastoma multiforme (GBM) is one of the most fatal forms of brain cancer, and patients with this disease develop resistance to chemotherapy and radiotherapy, which is associated with a poor patient prognosis $(1,2)$. Cisplatin is one of the first-line chemotherapeutic agents used to treat a number of human tumors, including GBM. It targets tumor cells by interacting with and damaging DNA, which induces apoptosis-mediated cell death (3). Despite an initial response, repeated clinical treatment with cisplatin usually results in the development of chemoresistance in tumor cells $(4,5)$. Therefore, investigating the molecular mechanisms underlying cisplatin-resistance and identifying efficient combination treatments to eliminate cisplatin resistance, are urgently required for the development of effective therapeutic strategies for GBM.

The long non-coding RNA (lncRNA), maternally expressed gene 3 (MEG3), is an imprinted gene that is part of the $\Delta$-like non-canonical notch ligand 1-MEG3 locus located on human chromosome 14q32 (6). Although MEG3 is expressed in a number of normal human tissues, particularly those of the brain and pituitary, loss of MEG3 expression has been identified in several human cancers, including bladder cancer, glioma, hepatocellular carcinoma and additional cancers (7-10). In addition, MEG3 was reported to be downregulated in human meningioma when compared with normal tissues, and was associated with tumor grade $(8,11)$. A Previous study demonstrated that loss of MEG3 activated autophagy in bladder cancer (9). However, the effects of MEG3 on cisplatin resistance and autophagy in GBM remain elusive.

In the present study, MEG3 expression was induced by cisplatin treatment of U87 cells, and increased MEG3 expression contributed to the induction of apoptosis. In addition, overexpression of MEG3 eliminated cisplatin-induced autophagy in U87 cells. Furthermore, inhibition of autophagy by MEG3 enhanced cisplatin-induced apoptosis in U87 cells. Therefore, the results present a novel therapeutic strategy for the treatment of GBM.

\section{Materials and methods}

Cell culture. The human U87 cell line, which is likely to be a glioblastoma cell line of unknown origin (12), was obtained 
from the American Type Culture Collection (Manassas, VA, USA). U87 cells were cultured at $37^{\circ} \mathrm{C}$ and $95 \%$ humidity in minimum essential medium (cat. no. M2297; Sigma-Aldrich; Merck KGaA, Darmstadt, Germany) containing 10\% fetal bovine serum (FBS; Gibco; Thermo Fisher Scientific, Inc., Waltham, MA, USA). The medium was refreshed every other day, and cells were passaged prior to reaching $100 \%$ confluence.

Reagents. The following primary antibodies were employed in the present study: Anti-caspase 3 (cat. no. 9662; dilution, 1:1,000; rabbit; Cell Signaling Technology, Inc., Danvers, MA, USA); anti-GAPDH (cat. no. SC-25778; dilution, 1:2,000; rabbit; Santa Cruz Biotechnology, Inc., Dallas, TX, USA); anti-poly (ADP) ribose polymerase (PARP; cat. no. SC-8007; dilution, 1:1,000; mouse; Santa Cruz Biotechnology, Inc.); anti-microtubule-associated proteins $1 \mathrm{~A} / 1 \mathrm{~B}$ light chain 3A (LC3; cat. no. L7543; dilution, 1:1,000; rabbit; Sigma-Aldrich; Merck KGaA); anti-autophagy protein 5 (ATG5; cat. no. sc-133158; dilution, 1:500; mouse; Santa Cruz Biotechnology, Inc.) and anti-p62 (cat. no. ab109012; dilution, 1:1,000; rabbit; Abcam, Cambridge, UK). The PARP antibody was used to detect PARP and cleaved PARP, the LC3 antibody was used to detect LC3 I and LC3 II, and the caspase 3 antibody was used to detect pro-caspase-3 and cleaved-caspase-3. Horseradish peroxidase-conjugated goat anti-mouse IgG (cat. no. A21010; dilution, 1:5,000) and anti-rabbit IgG (cat. no. A21020; dilution, 1:5,000) were purchased from Abbkine Scientific Co., Ltd. (Wuhan, China). Cisplatin was purchased from Sigma-Aldrich; Merck KGaA (cat. no. P4394), and the phosphoinositide 3-kinase (PI3K) inhibitor, 3-methyladenine (3-MA), was purchased from R\&D Systems, Inc. (Minneapolis, MN, USA). 3-MA inhibits autophagy by preventing autophagosome formation via the inhibition of PI3K catalytic subunit type 3 (13). 3-MA was resuspended in water, as organic solvents, such as dimethyl sulfoxide (DMSO), may be cytotoxic. Due to its instability in aqueous solutions, $50 \mathrm{mM}$ stocks of 3-MA were freshly prepared, and cells were treated with $5 \mathrm{mM} 3-\mathrm{MA}$ to inhibit autophagy.

Lentivirus transfection and MEG3 knockdown. The lentiviral transduction particles for short hairpin (sh)RNA-mediated knockdown of ATG5 were purchased from Sigma-Aldrich; Merck KGaA. The shRNA sequence targeting ATG5 was 5'-CCTTTCATTCAGAAGCTGTTT-3' and the pLKO.1 vector (Sigma-Aldrich; Merck $\mathrm{KGaA}$ ) was used as negative control. The shRNA was cloned using the PLKO.1 vector. Stable knockdown of ATG5 in cells was achieved as previously described (14). The primer sequences, 5'-AGACGGCGGAGA GCAGAG-3' and 5'-CACATTTATTGAGAGCACAGTGG-3', were used to generate plasmids encoding the full-length MEG3 sequence. RNA was extracted from U87 cells using RNAiso Plus (Takara Biotechnology Co., Ltd., Dalian, China) MEG3 cDNA was then reverse transcribed using $100 \mu \mathrm{g}$ total RNA from U87 cells and the Advantage RT-For PCR kit (Takara Biotechnology Co., Ltd.), and was amplified for 40 cycles of $95^{\circ} \mathrm{C}$ for $1 \mathrm{~min}, 54^{\circ} \mathrm{C}$ for $1 \mathrm{~min}$ and $72^{\circ} \mathrm{C}$ for $30 \mathrm{sec}$. To generate lentiviral vectors expressing MEG3, HEK293T cells (1x10 $/$ well) cultured on a $6-\mathrm{cm}$ dish were transfected with $2 \mu \mathrm{g}$ pCDH-MEG3 or pCDH vector (System Biosciences, Inc.,
Palo Alto, CA, USA), together with $1.5 \mu \mathrm{g}$ psPax2 (System Biosciences, Inc.) and $0.5 \mu \mathrm{g}$ pMD2 G (System Biosciences, Inc.) vectors using Lipofectamine 3000 (Thermo Fisher Scientific, Inc.). At $24 \mathrm{~h}$ following transfection, the medium was replaced with Dulbecco's modified Eagle's medium containing $10 \%$ FBS, and cells were incubated for a further $24 \mathrm{~h}$. The culture medium containing lentiviral particles was centrifuged at $1,000 \mathrm{x}$ for $5 \mathrm{~min}$ at room temperature. Viral particles were collected in the supernatant and the concentration was determined using the Lenti-Pac ${ }^{\mathrm{TM}}$ Lentivirus Concentration Solution (GeneCopoeia, Inc., Rockville, MD, USA). The virus titer was determined by calculating the multiplicity of infection. For infection of U87 cells $\left(1 \times 10^{5}\right), 100 \mu 1$ viral particles $\left(1 \times 10^{8} / \mathrm{ml}\right)$ were used for $12 \mathrm{~h}$. In order to establish a stable cell line, at $24 \mathrm{~h}$ after infection was performed, $1 \times 10^{5}$ cells that were infected by viral particles $\left(1 \times 10^{8} / \mathrm{ml}\right)$ at $37^{\circ} \mathrm{C}$ were seeded in a 6 -well plate and puromycin $(5 \mu \mathrm{M})$ was used as a selection marker for the infected cells for $24 \mathrm{~h}$ at $37^{\circ} \mathrm{C}$. The expression efficiency was evaluated by western blot analysis.

For knockdown of MEG3 in U87 cells, small interfering (si)RNA molecules were custom designed and synthesized by Sigma-Aldrich; Merck KGaA. The sequences of the siRNAs were as follows: Control, 5'-TAC GTCCAAGGTCGGGCAGGAAGA-3'; and MEG3, 5'-GACU UAAACCAAUGCCCUA-3'. This was transfected into U87 cells (2x10 $/$ well) for $24 \mathrm{~h}$ using Lipofectamine 3000 and, at $36 \mathrm{~h}$ after transfection, cells were treated with $10 \mu \mathrm{M}$ cisplatin for 0 or $36 \mathrm{~h}$. For certain experiments, cells with or without knockdown of MEG3 were pretreated with $5 \mathrm{mM}$ 3-MA or mock treatment (water) at $36 \mathrm{~h}$ after transfection for $6 \mathrm{~h}$ at $37^{\circ} \mathrm{C}$ to inhibit autophagy, immediately prior to treatment with $10 \mu \mathrm{M}$ cisplatin for 0 or $36 \mathrm{~h}$.

Western blot analysis. U87 cells (1x105/well) were seeded into the six well cell culture plate and subjected to various treatments as described in the above paragraphs. Total protein extracted from U87 cells using radioimmunoprecipitation assay lysis buffer (Shanghai Qcbio Science and Technologies Co., Ltd., Shanghai, China) was used for immunoblotting. Briefly, cell lysates were centrifuged at 9,000 x g for $10 \mathrm{~min}$ at $4^{\circ} \mathrm{C}$, and the supernatant was collected. The protein concentration was determined using a BCA Protein assay kit (Pierce; Thermo Fisher Scientific, Inc.). Total protein (30-60 $\mu \mathrm{g}$ ) was separated on a $12 \%$ SDS-PAGE mini-gel, followed by transfer to a nitrocellulose membrane. The membranes were blocked with $5 \%$ fat-free dry milk in a Tris-buffered saline plus Tween-20 solution, consisting of $50 \mathrm{mM}$ Tris- $\mathrm{HCl}, 0.15 \mathrm{M} \mathrm{NaCl}$ and $0.1 \%$ Tween-20 (pH 7.5), overnight at $4^{\circ} \mathrm{C}$. Membranes were then incubated with primary antibodies overnight at $4^{\circ} \mathrm{C}$ and incubated with the secondary antibodies $(1: 5,000)$ at room temperature for $1 \mathrm{~h}$. An enhanced chemiluminescence detection system (GE Healthcare, Chicago, IL, USA) was used to visualize the expression of target proteins. Three samples from each group were analyzed, and the results were quantified using the Gel-Pro analyzer software (version, 4.0, GE Healthcare).

Cell viability. U87 cells (2x10//well) were seeded in 96-well plates and incubated for $24 \mathrm{~h}$. Cells were then treated with $10 \mu \mathrm{M}$ cisplatin for $36 \mathrm{~h}$ at $37^{\circ} \mathrm{C}$. MTT reagent $(0.5 \mathrm{mg} / \mathrm{ml}$; 
A

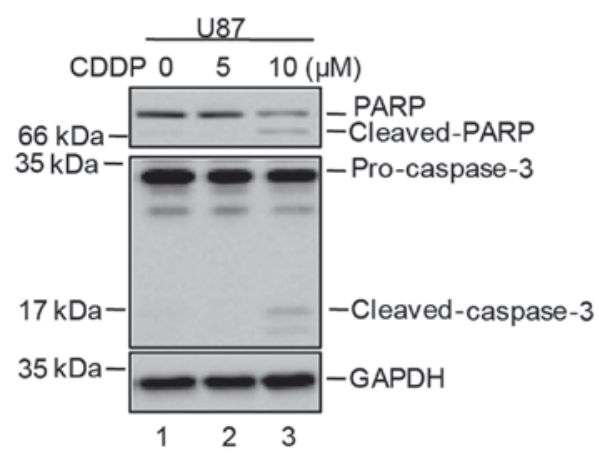

C

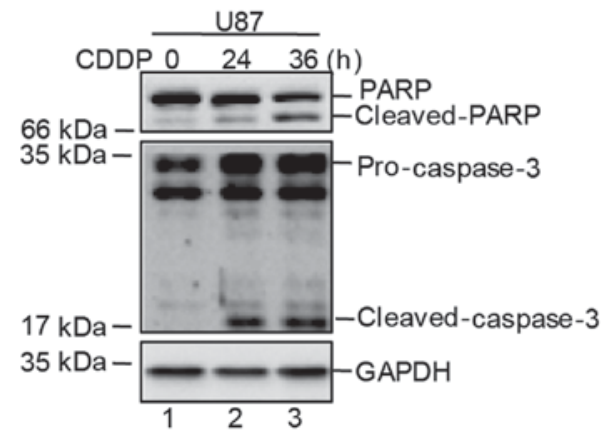

B

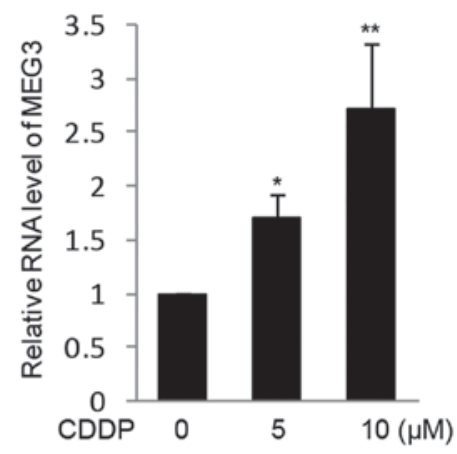

D

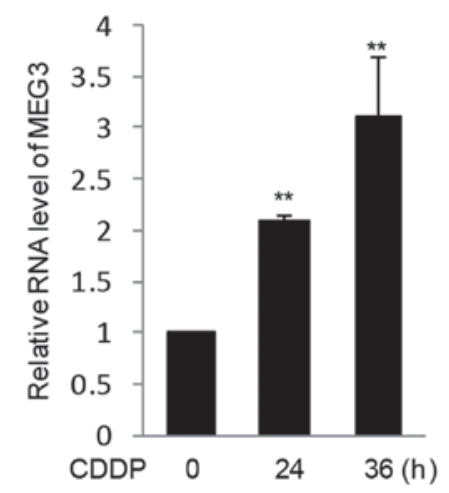

Figure 1. MEG3 expression is induced by CDDP treatment of U87 cells. U87 cells were treated with 0,5 and $10 \mu \mathrm{M}$ CDDP for $36 \mathrm{~h}$, and the (A) protein expression levels of apoptosis-associated factors and (B) MEG3 mRNA levels were determined. ${ }^{*} \mathrm{P}<0.05$ and ${ }^{* *} \mathrm{P}<0.01$ vs. $0 \mu \mathrm{M}$ CDDP. U87 cells were treated with $10 \mu \mathrm{M} \mathrm{CDDP}$ for 0,24 or $36 \mathrm{~h}$, and the (C) protein expression levels of apoptosis-associated factors and (D) MEG3 mRNA levels were analyzed. ${ }^{* * *} \mathrm{P}<0.01$ vs. 0 h. MEG3, maternally expressed gene 3; CDDP, cisplatin; PARP, poly(ADP) ribose polymerase.

Cayman Chemical Company, Ann Arbor, MI, USA) was subsequently added, and cells were incubated for $4 \mathrm{~h}$ before the medium was removed and $100 \mu \mathrm{l}$ DMSO was added. Cell viability was quantified by measuring the optical density (OD) at $490 \mathrm{~nm}$ using a microplate reader (Bio-Rad Laboratories, Inc., Hercules, CA, USA). At least three independent experiments were performed, and the final inhibitory rate of cell viability was calculated using the following formula: Inhibitory rate $=\left[1-\left(\mathrm{OD} 490_{\text {compound }} / \mathrm{OD} 490_{\text {solvent }}\right)\right] \times 100$.

Annexin V-fluorescein isothiocyanate (FITC) staining and fluorescence-activated cell sorting (FACS). The staining protocol was performed using a FITC Annexin V Apoptosis Detection kit I (BD Biosciences, Franklin Lakes, NJ, USA), according to the manufacturer's protocol. Briefly, $5 \times 10^{5}$ cells were transfected with $100 \mu \mathrm{l} \mathrm{pLKO} .1\left(1 \times 10^{8} / \mathrm{ml}\right)$ as a control or $100 \mu 1$ shRNA ATG5 $\left(1 \times 10^{8} / \mathrm{ml}\right)$ for $12 \mathrm{~h}$ at $37^{\circ} \mathrm{C}$ and, immediately following infection, the cells were transfected with control or MEG3 siRNA at $37^{\circ} \mathrm{C}$. After transfection for $24 \mathrm{~h}$, the cells were treated with $10 \mu \mathrm{M}$ cisplatin for $36 \mathrm{~h}$ at $37^{\circ} \mathrm{C}$. Cells were subsequently centrifuged at $1,000 \mathrm{x} \mathrm{g}$ for $5 \mathrm{~min}$ at $4^{\circ} \mathrm{C}$ and resuspended in $195 \mu \mathrm{l}$ binding buffer. This was followed by incubation with $5 \mu \mathrm{l}$ Annexin V-FITC for $10 \mathrm{~min}$ at room temperature in the dark. The cells were centrifuged again at $1,000 \mathrm{x}$ g for $5 \mathrm{~min}$ at $4^{\circ} \mathrm{C}$, before they were resuspended in $190 \mu \mathrm{l}$ binding buffer and $10 \mu \mathrm{l}$ propidium iodide stain and gently agitated. FACS analysis was performed using the BD Accuri C6 Flow Cytometer
(BD Biosciences) in order to detect cell apoptosis and the apoptosis was analyzed using CFlow Plus software (version, 1.0264.15; BD Biosciences).

RNA extraction and reverse transcription-quantitative polymerase chain reaction $(R T-q P C R)$. U87 cells $\left(2 \times 10^{5} /\right.$ well $)$ were treated with 5 or $10 \mu \mathrm{M}$ cisplatin for 0,24 or $36 \mathrm{~h}$, prior to RNA extraction using RNAiso Plus. RNA quality was examined by gel electrophoresis, and only high quality RNA was used for subsequent analyses. A total of $1 \mu \mathrm{g}$ total RNA was used to synthesize cDNA using the PrimeScript ${ }^{\mathrm{TM}}$ RT reagent kit (Perfect Real Time; Takara Biotechnology Co., Ltd.) according to the manufacturer's instructions. qPCR was performed using SYBR premix ExTaq (Takara Biotechnology Co., Ltd.) and ROX, and amplified with the Stratagene Mx3000P qPCR system (Agilent Technologies, Inc., Santa Clara, CA, USA). The primer sequences used for qPCR analysis were as follows: Actin forward, 5'-CTCCATCCTGGCCTCGC TGT-3', and reverse, 5'-GCTGTCACCTTCACCGTTCC-3'; MEG3 forward, 5'-GCATTAAGCCCTGACCTTTG-3', and reverse, 5'-TCCAGTTTGCTAGCAGGTGA-3'. The mRNA expression was normalized to the expression of actin using the $2^{-\Delta \Delta C q}$ method (15).

Statistical analysis. Data are presented as the mean \pm standard deviation, and statistical analysis was performed using SPSS software (version, 22.0; IBM Corp., Armonk, NY, USA). Statistical analysis was performed using one-way analysis of 
A

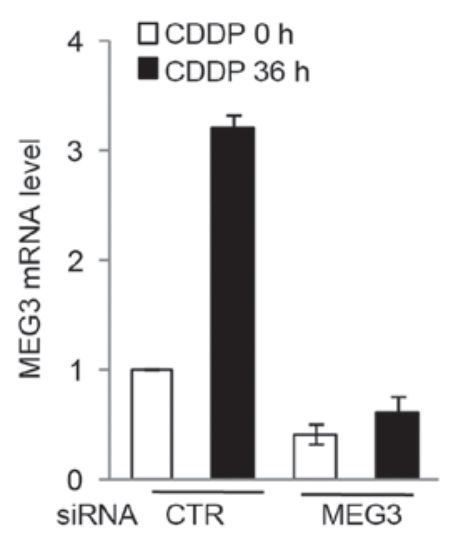

C

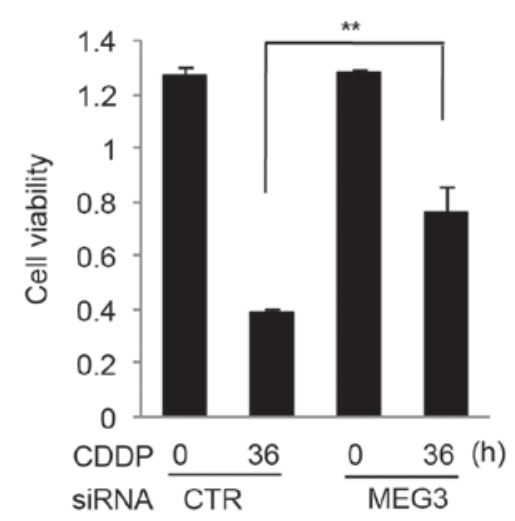

E

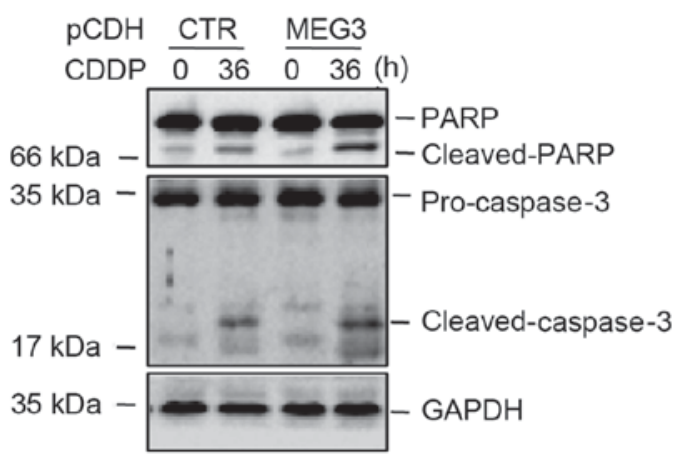

B

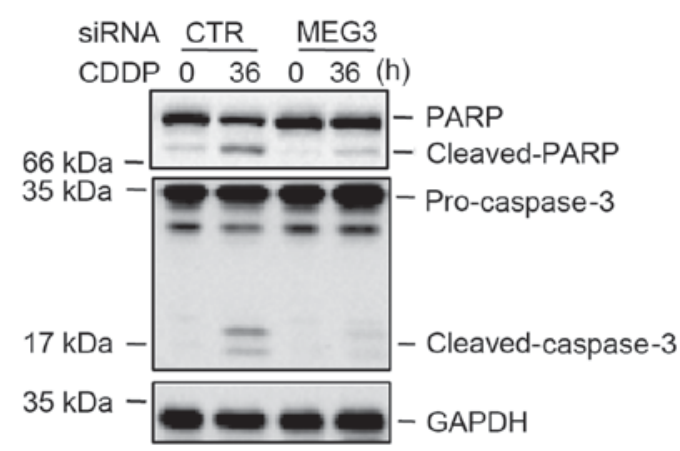

D

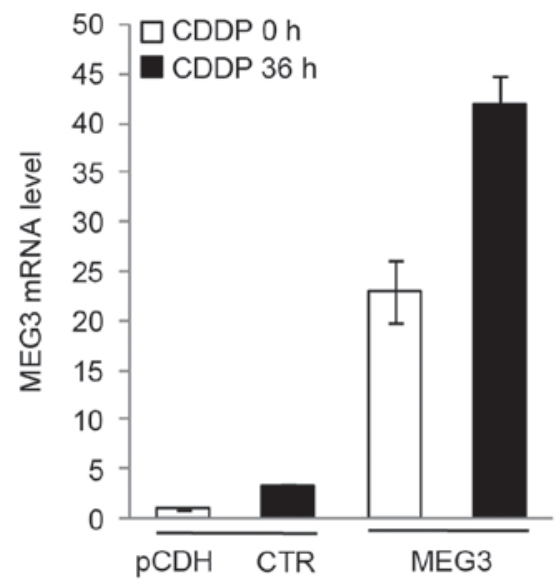

$\mathrm{F}$

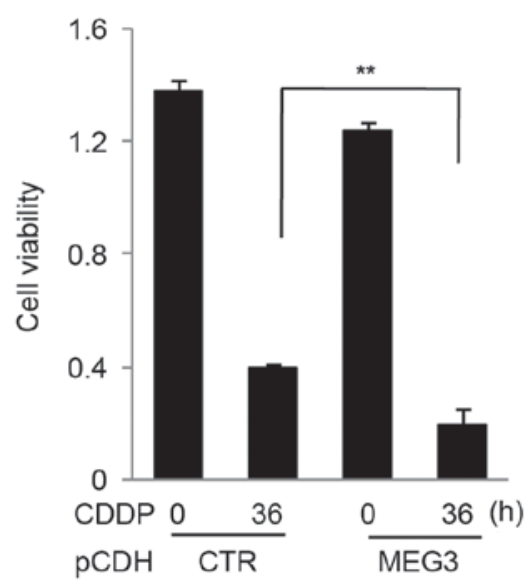

Figure 2. MEG3 enhanced CDDP-induced apoptosis in U87 cells. U87 cells were transfected with CTR or MEG3 siRNA. Following $24 \mathrm{~h}$, the cells were treated with $10 \mu \mathrm{M}$ CDDP for 0 or $36 \mathrm{~h}$. (A) MEG3 mRNA levels were analyzed by RT-qPCR. (B) Cell lysates were separated by SDS-PAGE and the expression levels of apoptosis-associated proteins were determined by western blotting. GAPDH was used as a loading control. (C) Cell viability was determined using an MTT assay. U87 cells transfected with CTR or MEG3 plasmids were treated with $10 \mu \mathrm{M}$ CDDP for 0 or $36 \mathrm{~h}$. (D) MEG3 mRNA levels were analyzed by RT-qPCR. (E) Cell lysates were separated by SDS-PAGE and the expression levels of apoptosis-associated proteins were determined by western blotting. GAPDH was used as a loading control. (F) Cell viability was determined using an MTT assay. Data are expressed as the mean \pm standard deviation (n=3). ${ }^{* *} \mathrm{P}<0.01$ as indicated. MEG3, maternally expressed gene 3; CTR, control; siRNA, small-interfering RNA; CDDP, cisplatin; RT-qPCR, reverse transcription-quantitative polymerase chain reaction; PARP, poly(ADP) ribose polymerase.

variance followed by Dunnett's test. $\mathrm{P}<0.05$ was considered to indicate a statistically significant difference.

\section{Results}

MEG3 IncRNA is induced by cisplatin treatment of human U87 cells. In order to evaluate MEG3 expression in response to cisplatin treatment of GBM, human U87 cells were treated with 0,5 or $10 \mu \mathrm{M}$ cisplatin for $36 \mathrm{~h}$. As shown in Fig. 1A, U87 cells were treated with 0,5 or $10 \mu \mathrm{M}$ cisplatin for $24 \mathrm{~h}$ and cell apoptosis was increased at $10 \mu \mathrm{M}$ cisplatin as indicated by increased PARP and caspase- 3 cleavage. Subsequently, the mRNA expression of MEG3 was detected using RT-qPCR and the results demonstrated that the mRNA expression levels of 
A
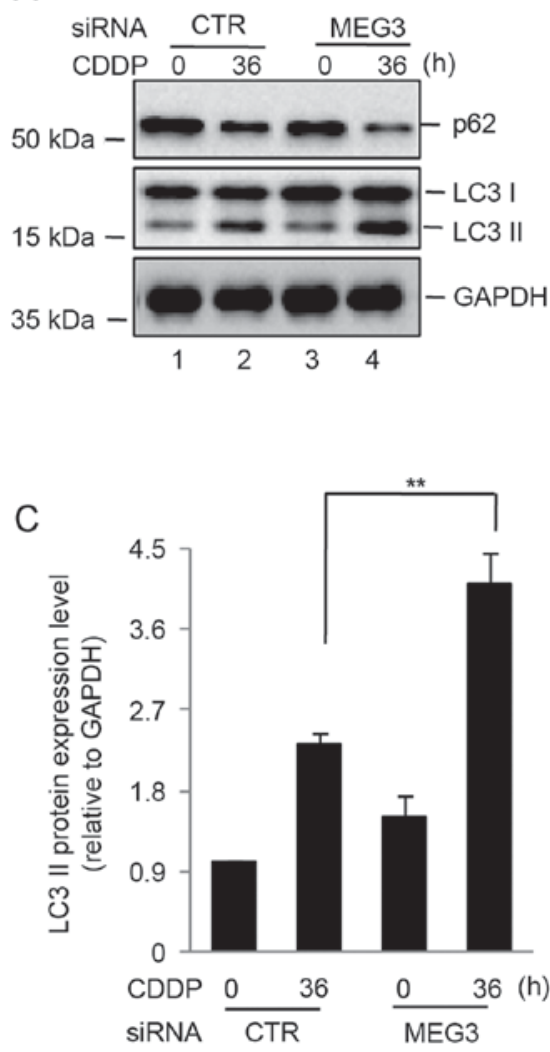

E

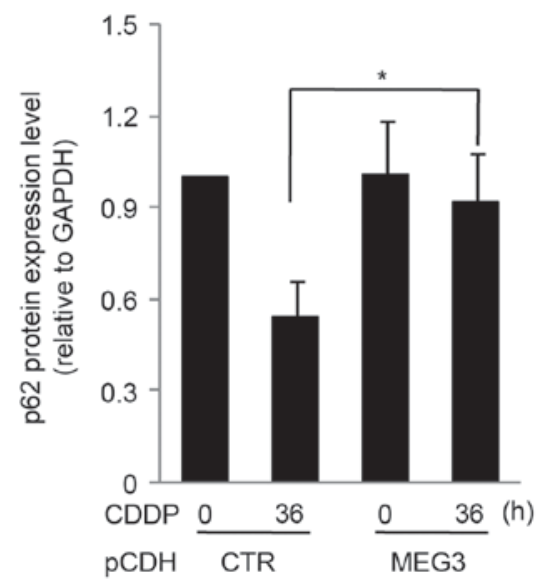

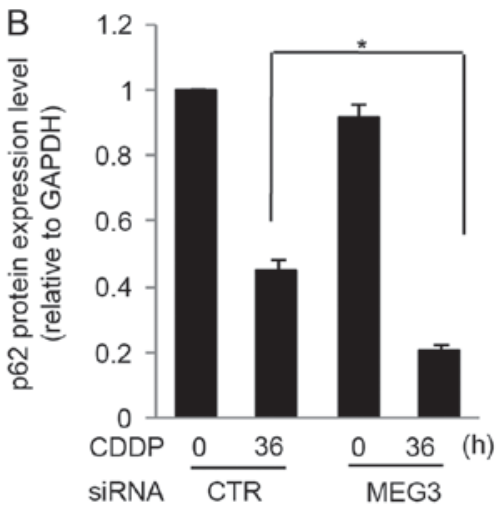

D

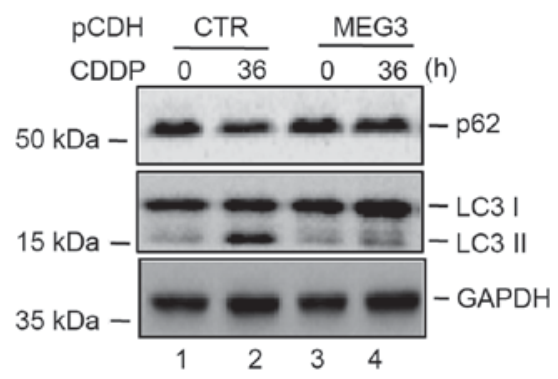

F

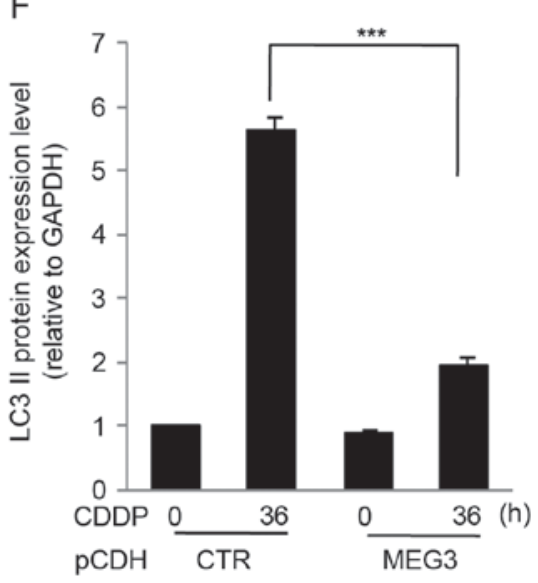

Figure 3. MEG3 decreased autophagy induced by CDDP. U87 cells transfected with CTR or MEG3 siRNA were treated with $10 \mu \mathrm{M}$ CDDP for 0 or $36 \mathrm{~h}$. (A) Cell lysates were separated by SDS-PAGE and the expression of autophagy-associated proteins was determined by western blot analysis. GAPDH was used as a loading control. Quantitative analysis of (B) p62 and (C) LC3 II protein expression levels. U87 cells transfected with CTR or MEG3 expression plasmids were treated with $10 \mu \mathrm{M}$ CDDP for 0 or $36 \mathrm{~h}$. (D) Cell lysates were separated by SDS-PAGE and the expression of autophagy-associated proteins was determined by western blot analysis. GAPDH was used as a loading control. Quantitative analysis of (E) p62 and (F) LC3 II protein expression levels. ${ }^{*} \mathrm{P}<0.05,{ }^{* *} \mathrm{P}<0.01$ and ${ }^{* * * *} \mathrm{P}<0.001$ as indicated. MEG3, maternally expressed gene 3; CTR, control; siRNA, small-interfering RNA; CDDP, cisplatin; LC3, microtubule-associated proteins 1A/1B light chain 3A.

MEG3 were increased in a dose-dependent manner following treatment of U87 cells with cisplatin (Fig. 1B). The cells were subsequently treated with $10 \mu \mathrm{M}$ cisplatin for 0,24 or $36 \mathrm{~h}$. As shown in Fig. 1C, cell apoptosis was increased at 24 and $36 \mathrm{~h}$, as indicated by increased PARP and caspase- 3 cleavage. In addition, the expression levels of MEG3 were significantly upregulated in a time-dependent manner in U87 cells (Fig. 1D).

MEG3 IncRNA promotes cisplatin-induced apoptosis in U87 cells. To determine whether elevated MEG3 affected cell survival following cisplatin treatment, MEG3 expression was inhibited by siRNA in U87 cells. The expression level of MEG3 was markedly downregulated in MEG3-siRNA transfected U87 cells when compared with those transfected with control siRNA (Fig. 2A). The cells were subsequently treated with cisplatin for 0 or $36 \mathrm{~h}$. When compared with cells transfected with control siRNA, inhibition of MEG3 reduced the protein expression levels of cleaved PARP (Fig. 2B). Similarly, decreased MEG3 expression attenuated the cisplatin-induced reduction in cell viability (Fig. 2C). Conversely, exogenous 
A
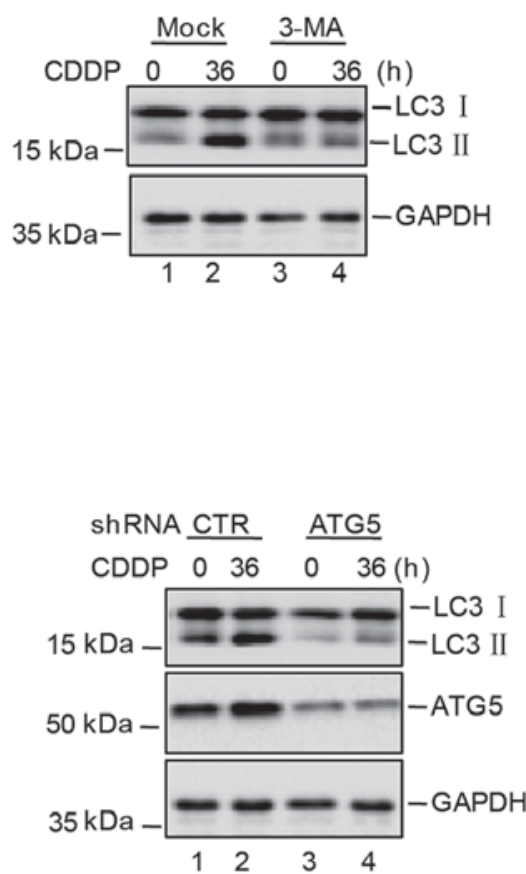

$\mathrm{E}$

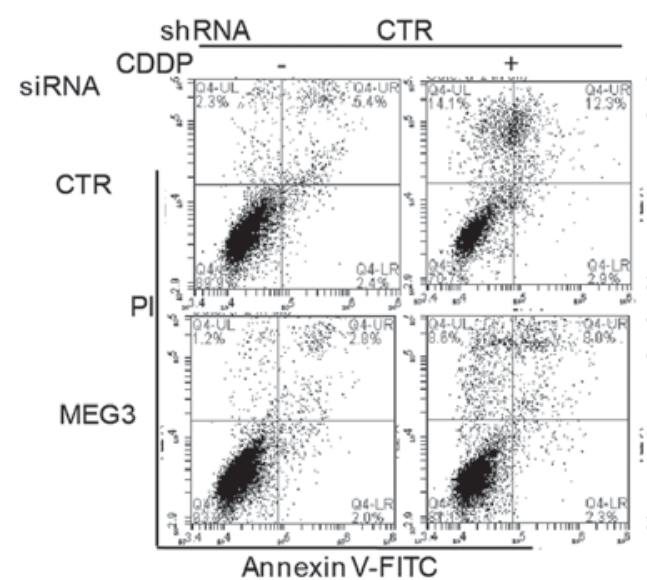

B

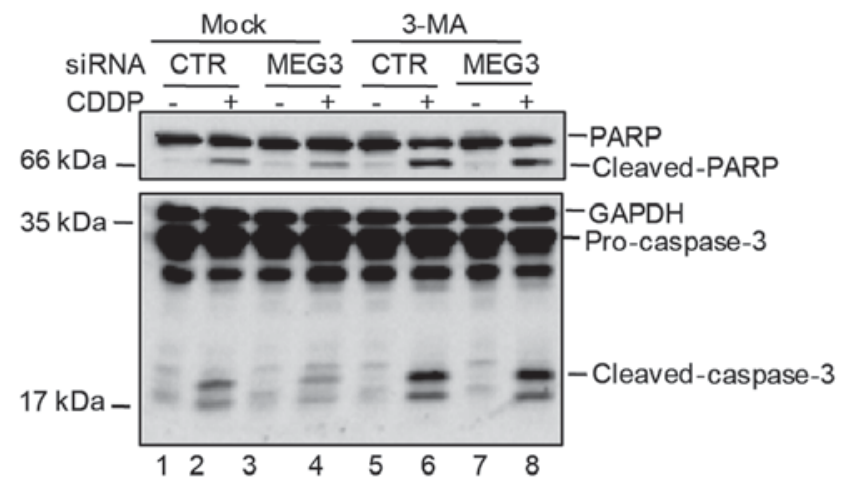

D

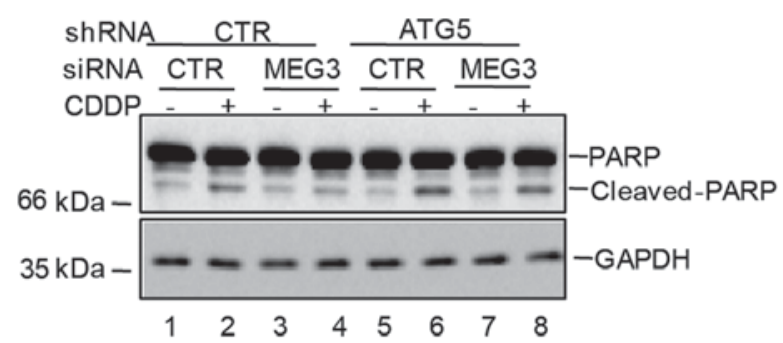

Figure 4. MEG3 promoted CDDP-induced apoptosis via inhibition of autophagy. (A) U87 cells were pretreated with or without 3-MA (5 mM) for $6 \mathrm{~h}$ and were subsequently treated with $10 \mu \mathrm{M}$ CDDP for 0 or $36 \mathrm{~h}$, and the expression of autophagy-associated proteins, LC3 I and II, were measured by western blotting. (B) U87 cells were transfected with CTR or MEG3 siRNA. At $36 \mathrm{~h}$ following transfection, the cells were pretreated with or without 3-MA (5 mM) for $6 \mathrm{~h}$ and were subsequently treated with $10 \mu \mathrm{M}$ CDDP for 0 or $36 \mathrm{~h}$. Cell lysates were separated by SDS-PAGE and the expression levels of apoptosis-associated factors were analyzed by western blotting. (C) Western blot analysis of the expression levels of autophagy-associated proteins and ATG5 in U87 cells following transfection with CTR or ATG5 shRNA and treatment with CDDP for 0 or $36 \mathrm{~h}$. U87 cells were then transfected with CTR or ATG5 shRNA, together with CTR or MEG3 siRNA. At $36 \mathrm{~h}$ following transfection, cells were treated with or without $10 \mu \mathrm{M}$ CDDP for 0 or $36 \mathrm{~h}$. (D) Cell lysates were separated by SDS-PAGE and the protein expression levels of PARP and cleaved-PARP were analyzed by western blotting. (E) The level of apoptosis in U87 cells transfected with CTR or ATG5 shRNA, together with CTR or MEG3 siRNA and treated with or without $10 \mu \mathrm{M}$ CDDP for 0 or $36 \mathrm{~h}$, was determined by flow cytometry analysis and (F) the results were quantified. The results are presented as the mean \pm standard deviation $(\mathrm{n}=3) .{ }^{* * * *} \mathrm{P}<0.001$ as indicated. MEG3, maternally expressed gene 3 ; CDDP, cisplatin; 3-MA, 3-methyladenine; LC3, microtubule-associated proteins 1A/1B light chain 3A; CTR, control; siRNA, small-interfering RNA; ATG5, autophagy protein 5; shRNA, short-hairpin RNA; PARP, poly(ADP) ribose polymerase; PI, propidium iodide; FITC, fluorescein isothiocyanate. expression of MEG3 efficiently enhanced the effects of cisplatin on the protein expression levels of cleaved PARP and cell viability (Fig. 2D-F).

MEG3 lncRNA inhibits cisplatin-induced autophagy. A previous study demonstrated that loss of MEG3 expression activated autophagy and increased the proliferation of bladder cancer cells (9). In order to investigate the effect of MEG3 on autophagy induced by cisplatin, U87 cells transfected with control MEG3 siRNA were treated with cisplatin for 0 or $36 \mathrm{~h}$. Compared with the control cells, silencing of MEG3 expression increased the protein expression of LC3 II relative to GAPDH and promoted p62 degradation (Fig. 3A-C). By contrast, overexpression of MEG3 attenuated cisplatin-induced autophagy in U87 cells (Fig. 3D-F).

LncRNA MEG3 enhances cisplatin-induced apoptosis via suppression of autophagy. Autophagy has been demonstrated 
to be a key mechanism that induces chemoresistance in cancer cells (9). Therefore, the present study investigated whether the effects of MEG3 in promoting cisplatin-induced apoptosis was dependent on the inhibition of autophagy. U87 cells transfected with control or MEG3 siRNA were pretreated with 3-MA (5 mM) for $6 \mathrm{~h}$ and exposed to $10 \mu \mathrm{M}$ cisplatin for 0 or $36 \mathrm{~h}$. Cell apoptosis was analyzed. Compared with control siRNA-transfected cells, inhibition of MEG3 markedly decreased the expression of apoptosis-associated proteins, which was reversed following treatment with the autophagy inhibitor, 3-MA (Fig. 4A and B). To confirm these observations, cells were infected with lentiviruses expressing shRNA targeting ATG5, which is a key molecule involved in the formation of autophagic vacuoles and induces autophagy (16). The cells were treated with control or MEG3 siRNA. When compared with control shRNA-transfected U87 cells that were transfected with MEG3 siRNA, knockdown of ATG5 significantly increased the level of apoptosis (Fig. 4C-F). These data indicated that inhibition of autophagy by MEG3 promoted cisplatin-induced apoptosis of U87 cells.

\section{Discussion}

LncRNAs, which were initially thought to be spurious transcriptional 'noise', have been reported to participate in a broad spectrum of biological processes, including maintenance of genome integrity, cell differentiation and embryonic development (17). In different types of cancer cells, lncRNAs have been demonstrated to be involved in tumorigenesis and chemoresistance (18).

The MEG3 lncRNA has been suggested to function as a tumor suppressor in a number of human cancers, and the expression level of MEG3 is significantly downregulated in several primary cancers $(6,8)$. A recent report indicated that MEG3 contributes to cisplatin-induced apoptosis in human lung cancer cells (19). Similarly, the results of the present study suggested that MEG3 expression was induced by cisplatin treatment of human U87 cells in a time- and dose-dependent manner. Silencing of MEG3 expression using siRNAs inhibited cisplatin-induced apoptosis in U87 cells. By contrast, exogenous MEG3 expression enhanced cisplatin-induced cell apoptosis.

Previous studies have revealed that autophagy is involved in mediating chemoresistance to cisplatin in several cancer cell types $(4,20)$. In the present study, MEG3 was observed to regulate cisplatin-induced autophagy in U87 cells, as inhibition of MEG3 expression promoted autophagy of cells treated with cisplatin. However, the molecular mechanisms underlying these effects remain unclear, and future studies will investigate this further. The results of the current study demonstrated that inhibition of autophagy by 3-MA or knockdown of ATG5 reversed the decrease in cell apoptosis caused by MEG3 knockdown in U87 cells treated with cisplatin. Therefore, the results suggest that MEG3 enhances cisplatin-induced apoptosis via inhibition of autophagy in U87 cells and, therefore, MEG3 maybe considered a novel therapeutic target to counteract cisplatin-resistant gliomas.

\section{Acknowledgements}

The present study was supported by the National Nature Science Foundation of China (grant nos. 81372714, 81672480 and 81603448).

\section{References}

1. Van Meir EG, Hadjipanayis CG, Norden AD, Shu HK, Wen PY and Olson JJ: Exciting new advances in neuro-oncology: The avenue to a cure for malignant glioma. CA Cancer J Clin 60: 166-193, 2010.

2. Wen PY and Kesari S: Malignant gliomas in adults. N Engl J Med 359: 492-507, 2008.

3. Kelland L: The resurgence of platinum-based cancer chemotherapy. Nat Rev Cancer 7: 573-584, 2007.

4. Liu X, Sun K, Wang H and Dai Y: Knockdown of retinoblastoma protein may sensitize glioma cells to cisplatin through inhibition of autophagy. Neurosci Lett 620: 137-142, 2016.

5. Wainwright DA, Nigam P, Thaci B, Dey M and Lesniak MS: Recent developments on immunotherapy for brain cancer. Expert Opin Emerg Drugs 17: 181-202, 2012.

6. Miyoshi N, Wagatsuma H, Wakana S, Shiroishi T, Nomura M, Aisaka K, Kohda T, Surani MA, Kaneko-Ishino T and Ishino F: Identification of an imprinted gene, Meg3/Gt12 and its human homologue MEG3, first mapped on mouse distal chromosome 12 and human chromosome 14q. Genes Cells 5: 211-220, 2000.

7. Anwar SL, Krech T, Hasemeier B, Schipper E, Schweitzer N, Vogel A, Kreipe $\mathrm{H}$ and Lehmann U: Loss of imprinting and allelic switching at the DLK1-MEG3 locus in human hepatocellular carcinoma. PloS One 7: e49462, 2012.

8. Balik V, Srovnal J, Sulla I, Kalita O, Foltanova T, Vaverka M, Hrabalek L and Hajduch M: MEG3: A novel long noncoding potentially tumour-suppressing RNA in meningiomas. J Neurooncol 112: 1-8, 2013.

9. Ying L, Huang Y, Chen H, Wang Y, Xia L, Chen Y, Liu Y and Qiu F: Downregulated MEG3 activates autophagy and increases cell proliferation in bladder cancer. Mol Biosyst 9: 407-411, 2013.

10. Zhou Y, Zhang X and Klibanski A: MEG3 noncoding RNA: A tumor suppressor. J Mol Endocrinol 48: R45-R53, 2012.

11. Zhang X, Gejman R, Mahta A, Zhong Y, Rice KA, Zhou Y, Cheunsuchon P, Louis DN and Klibanski A: Maternally expressed gene 3, an imprinted noncoding RNA gene, is associated with meningioma pathogenesis and progression. Cancer Res 70: 2350-2358, 2010.

12. Allen M, Bjerke M, Edlund H, Nelander S and Westermark B: Origin of the U87MG glioma cell line: Good news and bad news. Sci Transl Med 8: 354re3, 2016.

13. Miller S, Oleksy A, Perisic O and Williams RL: Finding a fitting shoe for Cinderella: Searching for an autophagy inhibitor. Autophagy 6: 805-807, 2010.

14. Han C, Gu H, Wang J, Lu W, Mei Y and Wu M: Regulation of L-threonine dehydrogenase in somatic cell reprogramming. Stem Cells 31: 953-965, 2013.

15. Livak KJ and Schmittgen TD: Analysis of relative gene expression data using real-time quantitative PCR and the 2(-Delta Delta C(T)) methods. Methods 25: 402-408, 2001.

16. Noda NN and Inagaki F: Mechanisms of autophagy. Annu Rev Biophys 44: 101-122, 2015.

17. Schmitt AM and Chang HY: Long noncoding RNAs in cancer pathways. Cancer Cell 29: 452-463, 2016.

18. Wu P, Zuo X, Deng H, Liu X, Liu L and Ji A: Roles of long noncoding RNAs in brain development, functional diversification and neurodegenerative diseases. Brain Res Bull 97: 69-80, 2013.

19. Liu J, Wan L, Lu K, Sun M, Pan X, Zhang P, Lu B, Liu G and Wang Z: The long noncoding RNA MEG3 contributes to cisplatin resistance of human lung adenocarcinoma. PLoS One 10: e0114586, 2015.

20. Yang X, Du T, Wang X, Zhang Y, Hu W, Du X, Miao L and Han C: IDH1, a CHOP and C/EBP $\beta$-responsive gene under ER stress, sensitizes human melanoma cells to hypoxia-induced apoptosis. Cancer Lett 365: 201-210, 2015. 